\title{
Spatio-temporal analysis to identify determinants of Oncomelania hupensis infection with Schistosoma japonicum in Jiangsu province, China
}

Kun Yang ${ }^{1,2^{*}}$, Wei Li $i^{1,2}$, Le-Ping Sun ${ }^{1,2}$, Yi-Xin Huang ${ }^{1,2}$, Jian-Feng Zhang ${ }^{1,2}$, Feng Wu ${ }^{1,2}$, De-Rong, Hang ${ }^{1,2}$, Peter Steinmann ${ }^{3,4}$ and You-Sheng Liang ${ }^{1,2}$

\begin{abstract}
Background: With the successful implementation of integrated measures for schistosomiasis japonica control, Jiangsu province has reached low-endemicity status. However, infected Oncomelania hupensis snails could still be found in certain locations along the Yangtze river until 2009, and there is concern that they might spread again, resulting in the possible re-emergence of infections among people and domestic animals alike. In order to establish a robust surveillance system that is able to detect the spread of infected snails at an early stage, sensitive and reliable methods to identify risk factors for the establishment of infected snails need to be developed.

Methods: A total of 107 villages reporting the persistent presence of infected snails were selected. Relevant data on the distribution of infected snails, and human and livestock infection status information for the years 2003 to 2008 were collected. Spatio-temporal pattern analysis including spatial autocorrelation, directional distribution and spatial error models were carried out to explore spatial correlations between infected snails and selected explanatory factors.

Results: The area where infected snails were found, as well as their density, decreased significantly between 2003 and 2008. Changes in human and livestock prevalences were less pronounced. Three statistically significant spatial autocorrelations for infected snails were identified. (i) The Moran's I of infected snails increased from 2004 to 2007, with the snail density increasing and the area with infected snails decreasing. (ii) The standard deviations of ellipses around infected snails were decreasing and the central points of the ellipses moved from West to East. (iii) The spatial error models indicated no significant correlation between the density of infected snails and selected risk factors.

Conclusions: We conclude that the contribution of local infection sources including humans and livestock to the distribution of infected snails might be relatively small and that snail control may limit infected snails to increasingly small areas ecologically most suitable for transmission. We provide a method to identify these areas and risk factors for persistent infected snail presence through spatio-temporal analysis, and a suggested framework, which could assist in designing evidence based control strategies for schistosomiasis japonica elimination.
\end{abstract}

Keywords: Schistosomiasis, Infected snails, Determinants, Spatio-temporal analysis, China

\footnotetext{
* Correspondence: jipdyk76@hotmail.com

1 Jiangsu Institute of Parasitic Diseases, Wuxi, China

${ }^{2}$ Key Laboratory on Technology for Parasitic Disease Prevention and Control,

Ministry of Health, Wuxi, China

Full list of author information is available at the end of the article
} 


\section{Background}

Schistosomiasis japonica is a zoonotic disease caused by an infection with Schistosoma japonicum. Oncomelania hupensis serves as the intermediate host snail of S. japonicum [1,2]. Previous studies have shown that O. hupensis in China is mainly distributed along the Yangtze river valley and in southern China. The distribution of S. japonicum is much more restricted [3]. Snails are infected when they are penetrated by miracidia, the larval stage of $S$. japonicum hatching from eggs when they reach water after being deposited with feces from the mammalian definitive hosts [4,5]. In the People's Republic of China, approximately 65 million individuals are currently at risk of infection with S. japonicum [6-8].

Jiangsu province is located on the lower reaches of the Yangtze river on the East coast of China (Figure 1). Flooding caused by the Yangtze river continues to be a prime risk factor for schistosomiasis in China $[9,10]$. It impacts on local disease endemicity, the number of acute cases and the geographic areas where infected snails are found as it increases their habitat [11-14]. In Jiangsu province, more than $90 \%$ of all current snail habitats are found along the shore of the Yangtze river. By the end of 2004, a total of 213,000 ha of habitat containing infected snails and 39 acute human schistosomiasis japonica cases were reported [10,15]. In 2005, the provincial government strengthened the implementation of integrated measures that aimed to reduce the transmission of S. japonicum [16]. In the wake of the programme, the average prevalence among humans decreased from $0.70 \%$ in 2005 to $0.11 \%$ in 2008 , and among livestock from $0.019 \%$ to 0 . Furthermore, no infected snails were found in the province from 2009 onwards. The surveillance system put in place to monitor the situation focuses mainly on humans and intermediate hosts. However, the determinants of infected snail occurrence requires further study in order to identify relevant risk factors and efficiently prevent the reemergence of infected snail populations. Several studies have demonstrated that snails were always present in clusters $[17,18]$. The application of spatial technology, including geographical information systems (GIS), remote sensing (RS) and spatial statistics for schistosomiasis research since the 1990s has resulted in important advances in our understanding of the key factors determining schistosomiasis transmission [2,19-21]. Recently, the application of directional distribution analysis became more common in many study fields, for example the mapping of crime hotspots where it might identify a relationship between the distribution and trends of criminal activity and particular physical attributes (e.g. a particular street section) [22].

In the study presented here, we analyze the relationship between infected snail populations and local infection sources, including people and livestock in Jiangsu province based on spatio-temporal - including directional distribution - analysis, to identify the main source of infection, and explore the environmental determinants of $O$. hupensis infection.

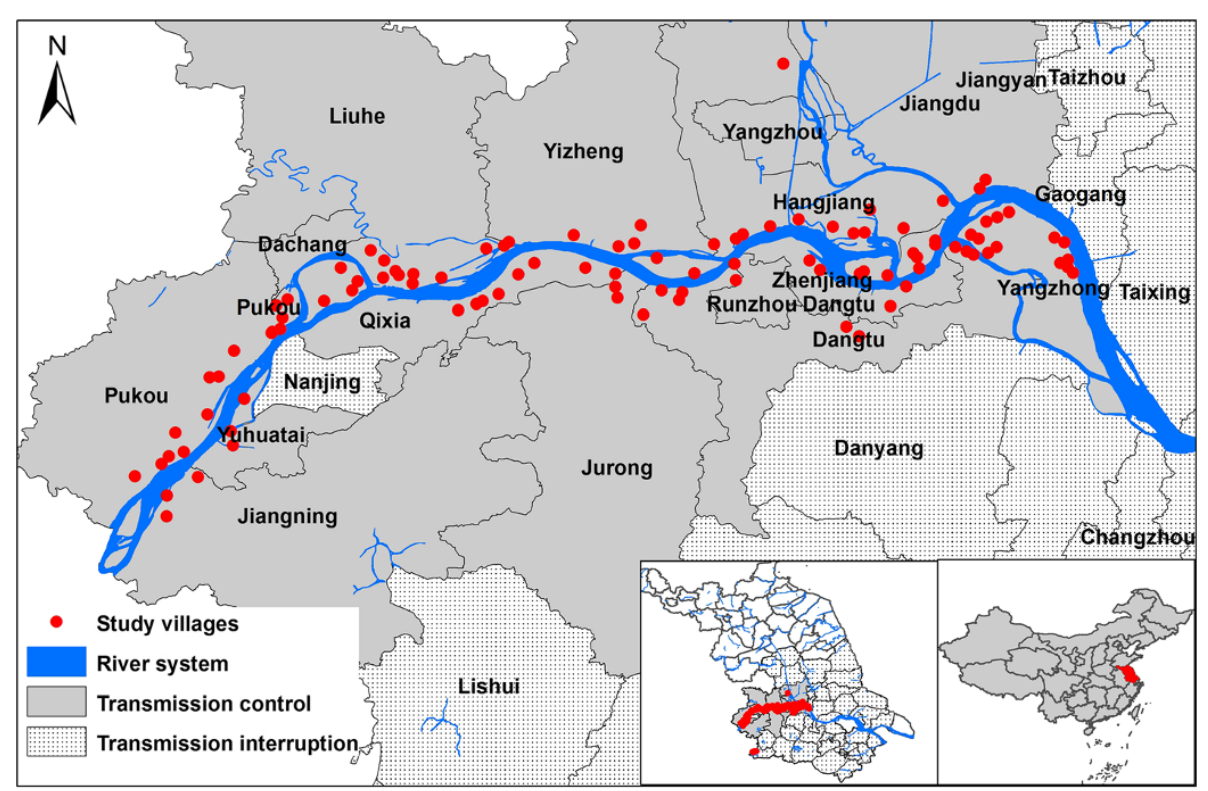

Figure 1 Location of the study area in Jiangsu province, China, and distribution of study villages. The schistosomiasis japonica control situation is described as transmission interruption (no snail is found) or transmission control (no acute case of schistosomiasis is found). 


\section{Methods}

\section{Study area}

The study focused on the marshland along the Yangtze river in Jiangsu province where 107 villages (Figure 1) were selected based on the following criterion: at least one infected snail had been found around the village, including in marshlands along the Yangtze river and on beaches of the rivers connected to the Yangtze river, between 2003 and 2008. The geographical co-ordinates (latitude/longitude) of each village were recorded by a GPS unit (Garmin Map76).

Between 2003 and 2008, annual surveillance covering both the human population and livestock had been carried out within the study area. In each of the study villages, all heads of livestock were examined using the standard miracidia hatching method [23]. Among humans, $90 \%$ of the individuals aged between 6 and 60 years were screened for schistosomiasis japonica infection using a serological test (Dipstick dye immunoassay, DDIA) [24]. Stool samples were then collected from individuals with positive test results to conduct the KatoKatz thick smear test [23]. A single dose of praziquantel at a dosage of $40 \mathrm{mg} / \mathrm{kg}$ body weight was offered to all seropositive individuals, and a two-day course of praziquantel at $60 \mathrm{mg} / \mathrm{kg}$ body weight was administered to those with a positive Kato-Katz thick smear test.

Snail collection was conducted by systematically sampling with a square frame of $0.11 \mathrm{~m}^{2}$ that was set every 30 meters in known snail habitats. All snails inside the frame were collected. Additionally, environmental (purposeful) sampling was employed in spring and autumn of each year to detect snails in potential snail habitats in grasslands and marshlands, e.g. where snails had been detected over the last three years, in previously flooded areas etc. Systematic sampling was then carried out if any snails were found in these potential habitats. All collected snails were counted, crushed and examined by microscopy to detect sporocysts and cercariae. Various outcome indices were considered, including the $S$. japonicum prevalence among humans, the rate of S. japonicum infection in snails, the density of living snails, and the density of infected snails.

\section{Statistical analysis}

Descriptive analysis was performed using the statistical software package SPSS (Version 11, SPSS Inc. Chicago, IL, USA). The analysis focused on the yearly data for $S$. japonicum infection among snails and the infection status of the human and livestock populations.

The spatio-temporal pattern analysis was carried out using the spatial analyst module of ArcGIS 10.0 (ESRI, Redlands, CA, USA) and GeoDA 1.0.1 (The GeoDa Center for Geospatial Analysis and Computation). The global Moran's I was used to measure spatial autocorrelation in infected snail, human and livestock populations in each year. The spatial autocorrelation was used to evaluate whether the pattern was clustered, dispersed, or random. A Z score was considered for evaluating the significance of the Moran's I value. The differences of spatial autocorrelation in each year were then used to explore spatio-temporal patterns.

Directional distribution, namely the Standard Deviational Ellipse (SDE), was used to measure the directional trend each year, and to provide information about dispersion of the infected snails, humans and livestock in terms of compactness and orientation. Employing the method was inspired by its wide application in diverse studies [25]. For example, plotting ellipses for a disease outbreak over time may be used to model its spread [26]. The distributional trend analysis can create an elliptical polygon; the attributed values for these output ellipse polygons include two standard distances (long and short axes) and the orientation of the ellipse. We used one standard deviation to represent the distribution that covers approximately 68 percent of all input variables for both the infected snails, humans and livestock [27,28]. A series of additional measurements and data including axial ratios, and coordinates of each ellipse in each year were collected to compare the spatial patterns of infected snails and local infection sources.

We used a spatial autoregressive error model (a spatial regression model including a spatial autoregressive error term) implemented in GeoDA 1.0.1 to measure the relationships between the density of infected snails and the serological or stool prevalence of people and livestock. Initially, we fit the data in an ordinary least squares (OLS) regression model. As expected, the results suggested considerable non-normality and heteroscedasticity, which did not satisfy the basic hypothesis of standard linear regression, as well as high spatial correlation. Based on this result we concluded that a spatial error model was more appropriate for this dataset.

Formally, this model is $y=X \beta+\varepsilon$, with $\varepsilon=\lambda W+\mu$, where $y$ is a vector of observations of the dependent variable, $W$ is the spatial weights matrix, $X$ is a matrix of observations of the explanatory variables, $\varepsilon$ is a vector of spatially auto correlated error terms, $\mu$ is a vector of i.i.d. errors, and $\lambda$ and $\beta$ are parameters.

\section{Ethics statement}

The study protocol was approved by the Ethics Review Committee of the Jiangsu Institute of Parasitic Diseases, Wuxi, China. Written informed consent had also been obtained from each participant or a literate relative during the screening for infections. No specific permits were required for the field studies focusing on snails as they did not involve endangered or protected species. 


\section{Results}

Table 1 summarizes the data related to infected snails. The area and density of infected snails were 1272.686 ha and 0.015 per $\mathrm{m}^{2}$ in 2003, which then decreased to 97.186 ha and 0.003 per $\mathrm{m}^{2}$ in 2008 , a decrease of $92.4 \%$ and $76.3 \%$, respectively. The human sero-prevalence was $1.649 \%$ and $1.304 \%$ in 2003 and 2008, respectively, a non-significant decrease $\left(\chi^{2}=7.538, \mathrm{df}=1, \mathrm{P}=0.06\right)$. The stool prevalence was $4.290 \%$ and $4.977 \%$ in 2003 and 2008, respectively, again not significantly different $\left(\chi^{2}=1.138, \mathrm{df}=1, \mathrm{P}=0.710\right)$. Among 4714 heads of livestock examined, 21 were positive, translating into a prevalence of $0.445 \%$ over the study period. No positive livestock was found in 2008. However, the difference was again not significant $\left(\chi^{2}=0.556, \mathrm{df}=1, \mathrm{P}=0.273\right)$.

The results of the global autocorrelation statistics for infected snails, human sero-prevalence, and human stool prevalence in each year are summarized in Table 2. The results of the global Moran's I tests were statistically significant (z-score greater than 1.96) and indicate spatial heterogeneity. The global autocorrelation statistics for infected snails in 2004 and 2008 and stool prevalence in 2004 were also statistically significant. The spatial autocorrelation is graphically depicted in Figure 2. Results show that the change in spatial autocorrelation of the sero-prevalence was relatively stable with intervals between -0.075 and 0.069. Between 2004 and 2007, the spatial correlation of the infected snail populations fell dramatically from 0.272 to 0.098 , and that of the stool prevalence also decreased from 0.342 to 0.063 . After 2007, a rapid increase was observed for the infected snails and a further decrease for the stool prevalence.

Figure 3 shows the series of directional distributions of the infected snails in each year. Their shapes are similar from one year to another, and the ellipses are generally oriented along the Yangtze river. From 2003 to 2008, both the long and short axes became shorter, which means that the standard deviations of the ellipses were decreasing. The central points of the ellipse polygons moved from West to East from 2003 to 2008.
Table 2 The yearly Moran's I value of S. japonicum-infected snails, sero-prevalence and stool prevalence in the study villages of Jiangsu province, China, from 2003 to 2008

\begin{tabular}{llll}
\hline Year & Infected snails & $\begin{array}{l}\text { Human sero- } \\
\text { prevalence }\end{array}$ & $\begin{array}{l}\text { Human stool } \\
\text { prevalence }\end{array}$ \\
\hline 2003 & 0.021 & 0.048 & -0.052 \\
2004 & $0.273^{*}$ & -0.045 & $0.342^{*}$ \\
2005 & 0.168 & -0.074 & 0.015 \\
2006 & 0.055 & 0.069 & 0.025 \\
2007 & 0.098 & -0.075 & 0.064 \\
2008 & $0.363 *$ & 0.014 & -0.048 \\
\hline
\end{tabular}

* $\mathrm{P}<0.05$.

The serial comparisons of the directional distribution from 2003 to 2008 are shown in Figure 4A-F. The ellipse polygons of the sero-prevalence retained a relatively stable shape while other polygons changed significantly. The shapes of these ellipse polygons were heterogeneous, with ellipse polygons intersecting in some regions but not in others. These patterns are not suggestive of a significant spatial relationship between infected snails and the investigated variables describing human infection status.

Table 3 presents the spatial error model estimates for the density of infected snails. The spatial regression results show that there was no significant correlation between the density of infected snails and other study factors $(P>0.05)$.

\section{Discussion}

The control of schistosomiasis japonica, similar to the control of any infectious disease, aims to interrupt the parasite lifecycle through interventions intended to eliminate the intermediate host, eliminate the parasite from the definitive host, prevent infection of the intermediate or definitive host, etc. [29,30]. In highly endemic regions, the provision of praziquantel to the local residents is effective at reducing the infection rate [31-33]. However, this does not always interrupt transmission, as throughout

Table 1 The characteristics of schistosomiasis japonica in the study villages of Jiangsu province, China, from 2003 to 2008

\begin{tabular}{|c|c|c|c|c|c|c|c|c|}
\hline \multirow[t]{2}{*}{ Year } & \multirow{2}{*}{$\begin{array}{l}\text { Area of infected } \\
\text { snail habitat (ha) }\end{array}$} & \multirow{2}{*}{$\begin{array}{l}\text { Density of infected } \\
\text { snails }(/ 0.1 \mathrm{~m} 2)\end{array}$} & \multicolumn{2}{|c|}{ Human sero-examination } & \multicolumn{2}{|c|}{ Human stool examination } & \multicolumn{2}{|c|}{ Livestock examination } \\
\hline & & & $\begin{array}{c}\text { No. } \\
\text { examined }\end{array}$ & $\begin{array}{l}\text { Prevalence } \\
(\%)\end{array}$ & $\begin{array}{c}\text { No. } \\
\text { examined }\end{array}$ & $\begin{array}{l}\text { Prevalence } \\
(\%)\end{array}$ & $\begin{array}{c}\text { No. } \\
\text { examined }\end{array}$ & $\begin{array}{c}\text { Prevalence } \\
\text { (\%) }\end{array}$ \\
\hline 2003 & 1272.686 & 0.849 & 18261 & 1.659 & 303 & 4.29 & 1001 & 0.3 \\
\hline 2004 & 1649.19 & 0.374 & 25750 & 4.726 & 1217 & 5.177 & 988 & 0.405 \\
\hline 2005 & 1110.31 & 0.438 & 27933 & 4.493 & 1255 & 1.673 & 834 & 0.959 \\
\hline 2006 & 758.051 & 0.616 & 42537 & 2.591 & 1102 & 2.904 & 641 & 0.624 \\
\hline 2007 & 516.948 & 0.263 & 43907 & 1.635 & 718 & 3.9 & 708 & 0.282 \\
\hline 2008 & 97.186 & 0.268 & 16940 & 1.305 & 221 & 4.977 & 542 & 0 \\
\hline
\end{tabular}




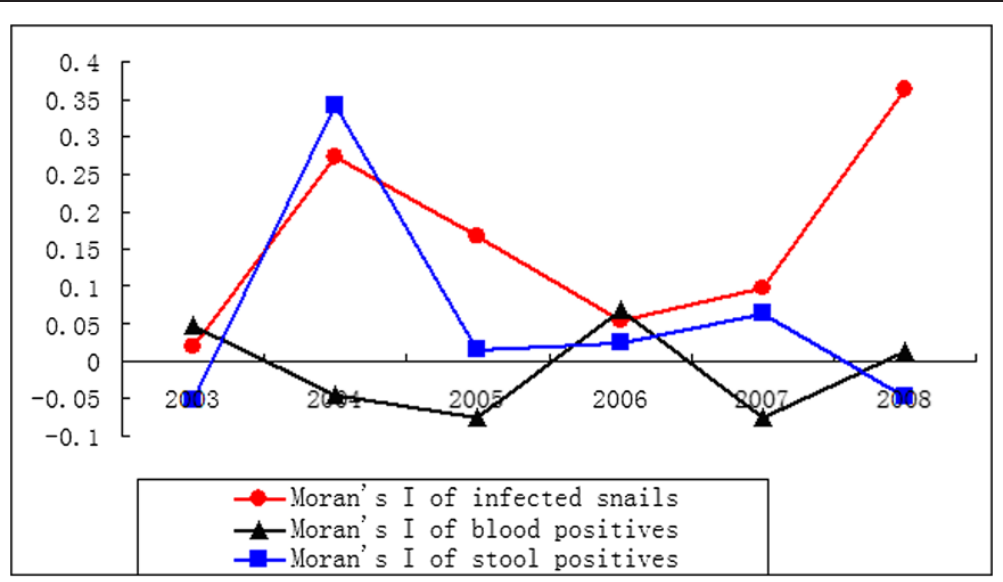

Figure 2 The dynamic change of Moran's I value of S. japonicum-infected snails, human sero-prevalence and human stool prevalence in the study villages of Jiangsu province, China, from 2003 to 2008.

history, livestock such as cattle were the main source of infection for schistosomiasis in many areas in China $[34,35]$. The transmission patterns and spatial distribution of the total and infected snails and the influences of environmental and socio-economic determinants have been considered in a series of epidemiological studies supported by spatial modeling [19].

In Jiangsu province, the area and density of infected snails decreased significantly until 2008. The number of livestock in endemic villages also decreased significantly, to 542 in 2008 from 1001 in 2003 . The prevalence in livestock was very low, with no infected livestock found in 2008. However, the prevalence in humans was stable, with no significant difference between serological and stool positive rates over the study period. This might indicate that the contribution from livestock to human infection is not as large anymore as it had been historically.
Moran's I (Spatial Statistics) measures spatial autocorrelation based on both locations and attribute information, and evaluates whether the pattern expressed is clustered, dispersed, or random [36,37]. In general, a Moran's Index value near +1.0 indicates clustering while an index value near -1.0 indicates dispersion. Figure 2 shows that the Moran's I of the sero-prevalence was between -0.075 and 0.069 , indicating that the distribution was random and the sero-prevalence stable. The Moran's I of infected snails increased from 2004 to 2007, indicating that the distribution of infected snails become more and more clustered in some regions. Indeed, the density increased from 0.373 to 0.616 infected snails per $0.1 \mathrm{~m}^{2}$, while the area with infected snails was decreasing dramatically. This suggests that schistosomiasis transmission is ongoing in certain areas and that control measures may be forcing transmission into ever smaller

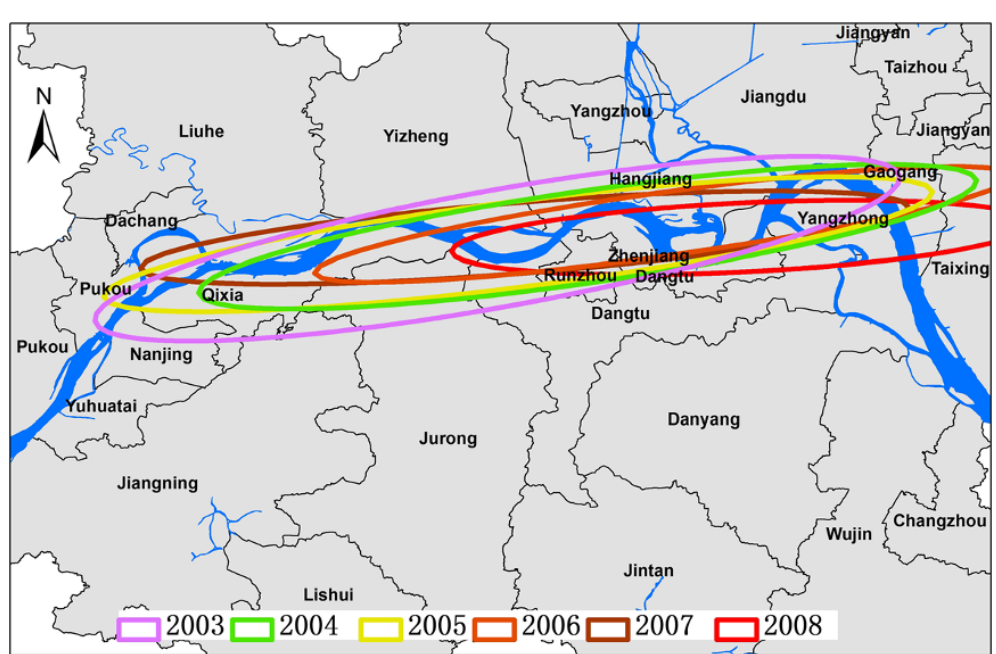

Figure 3 Directional distribution of S. japonicum-infected snails in Jiangsu province, China, from 2003 to 2008. 

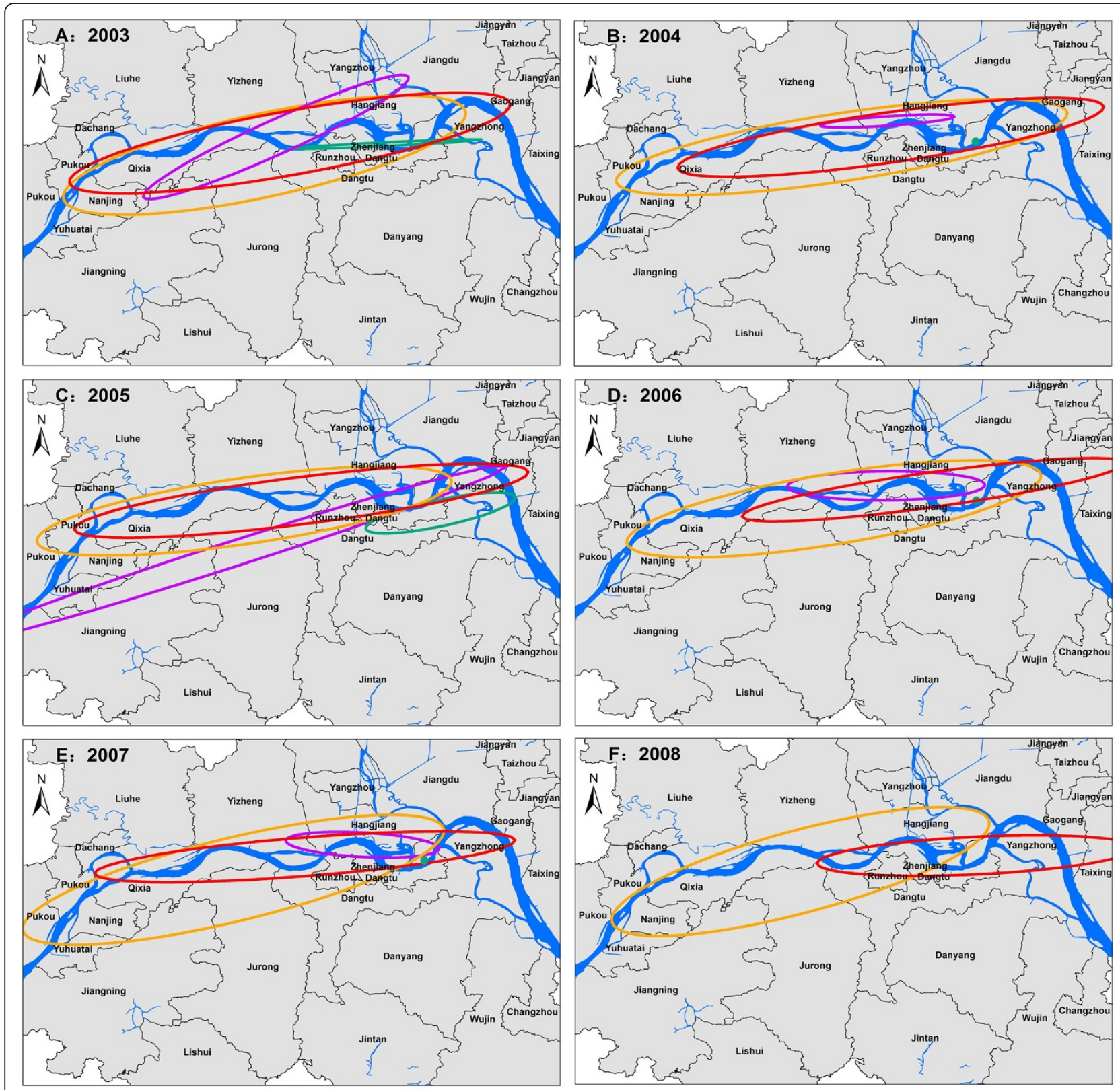

\section{Infected snails}

$\square$ Human stool positives

Human sero-positives

Figure 4 Directional distribution of S. japonicum-infected snails and study factors in the study villages of Jiangsu province, China, in each year from 2003 to 2008.

refugia (areas ecologically or otherwise most suitable for transmission). The variation curve of the stool prevalence also increased from 2004 to 2007. To determine whether these cluster regions were stable, the directional distribution analysis was carried out.

From 2003 to 2008, the standard deviations of ellipses around infected snail areas were decreasing and the central points of the ellipses moved from West to East, indicating that the habitats of infected snails had become smaller, and that clusters existed in special regions. The serial ellipse polygons in Figure 3A-F indicate that these spatial distributions were significantly different from each other, but the spatial correlation between infected snails and other study factors was not significant. The ellipse polygons overlap in some regions in each year, and studied factors appear to contribute to the distribution of infected snails in some regions. After integrating the spatial error model, we found that the relationship 
Table 3 Spatial error model estimations for the density of S. japonicum-infected snails in Jiangsu province, China, from 2003 to 2008

\begin{tabular}{ccccccc}
\hline Variable & $\mathbf{2 0 0 3}$ & $\mathbf{2 0 0 4}$ & $\mathbf{2 0 0 5}$ & $\mathbf{2 0 0 6}$ & $\mathbf{2 0 0 7}$ & $\mathbf{2 0 0 8}$ \\
\hline Constant & $0.032^{*}$ & $0.025^{*}$ & $0.019^{*}$ & $0.045^{*}$ & $0.024^{*}$ & $0.015^{*}$ \\
Human sero-prevalence & -0.051 & 0.177 & 0.001 & -0.444 & -0.178 & -0.195 \\
Human stool prevalence & -0.012 & -0.143 & -0.084 & -0.123 & -0.062 & -0.013 \\
Livestock prevalence & -0.062 & -0.609 & 0.034 & -0.015 & -0.215 & $-{ }^{* *}$ \\
LAMBDA & 0.026 & $0.423^{*}$ & $0.368^{*}$ & 0.008 & 0.167 & 0.002 \\
\hline
\end{tabular}

* $\mathrm{P}<0.05$.

** No infected livestock was found in 2008.

between the study factors and the spatial distribution of infected snails was not strong, confirming data from field studies. For example, no infected livestock or wild mice were detected in the infected snail habitats [38].

Limitations of the current study must also be recognized. First, the considered explanatory factors for infected snails were limited to the serological and parasitological status of the local human and livestock population, while the spatial distribution of infected snails may also depend on other factors, which consequently should be taken into account to improve model accuracy. Second, we explored the risk factors using retrospective data. Third, the sensitivity and specificity of serological and stool tests are not perfect $[39,40]$.

\section{Conclusions}

In conclusion, the contribution of the local infection sources including humans and livestock to the distribution of infected snails may not be significant, and external factors need further study, e.g. temporal migration from other endemic areas. It also appears that snail control may be restricting infected snails into smaller yet ecologically more suitable areas for transmission. The present study describes a way to identify risk factors through retrospective study and spatio-temporal analysis, and such a framework could assist in designing evidence-based control strategies in the process of schistosomiasis elimination.

\section{Competing interests}

The authors have declared that no competing interests exist.

\section{Authors' contributions}

Conceived and designed the experiments: KY YSL. Performed the experiments: KY WL LPS YXH JFZ FW DRH. Analyzed the data: KY WL. Contributed reagents/materials/analysis tools: KY WL. Wrote the paper: KY PS. All authors read and approved the final version of the manuscript.

\section{Acknowledgements}

The authors would like to acknowledge the staff at the local Center for Disease Control and Prevention Centers for their kind collaboration and making the field data available. This work received financial support from the National Natural Science Foundation of China (No. 81101275; 81101280), Natural Science Foundation of Jiangsu Province (BS2010153), Project of Public Health Department of Jiangsu Province (No. RC2011094), National S \&
T Major Program (No. 2012ZX10004-220), and was partially funded through a capacity building initiative for Ecohealth Research on Emerging Infectious Disease in Southeast Asia supported by the International Development Research Centre (IDRC).

\section{Author details}

${ }^{1}$ Jiangsu Institute of Parasitic Diseases, Wuxi, China. ${ }^{2}$ Key Laboratory on Technology for Parasitic Disease Prevention and Control, Ministry of Health, Wuxi, China. ${ }^{3}$ Department of Epidemiology and Public Health, Swiss Tropical and Public Health Institute, Basel, Switzerland. "University of Basel, Basel, Switzerland.

Received: 22 January 2013 Accepted: 4 May 2013

Published: 6 May 2013

\section{References}

1. Zhou XN, Bergquist R, Leonardo L, Yang GJ, Yang K, Sudomo M, Olveda R: Schistosomiasis japonica control and research needs. Adv Parasitol 2010, 72:145-178

2. Yang K, Zhou XN, Wu XH, Steinmann P, Wang XH, Yang GJ, Utzinger J, Li $\mathrm{HJ}$ : Landscape pattern analysis and Bayesian modeling for predicting Oncomelania hupensis distribution in Eryuan county, People's Republic of China. Am J Trop Med Hyg 2009, 81:416-423.

3. Lei ZL, Zheng H, Zhang L, Zhu R, Gao JG, Li SZ, Wang LY, Chen Z, Zhou XN: Schistosomiasis status in People's Republic of China in 2010 (in Chinese). Chin J Schisto Control 2011, 23:599-604.

4. Utzinger J, Zhou XN, Chen MG, Bergquist R: Conquering schistosomiasis in China: the long march. Acta Trop 2005, 96:69-96.

5. Wang LD, Utzinger J, Zhou XN: Schistosomiasis control: experiences and lessons from China. Lancet 2008, 372:1793-1795.

6. Zhou XN, Wang LY, Chen MG, Wu XH, Jiang QW, Chen XY, Zheng J, Utzinger J: The public health significance and control of schistosomiasis in China - then and now. Acta Trop 2005, 96:97-105.

7. Wang LD, Chen HG, Guo JG, Zeng XJ, Hong XL, Xiong JJ, Wu XH, Wang XH, Wang LY, Xia G, et al: A strategy to control transmission of Schistosoma japonicum in China. N Engl J Med 2009, 360:121-128.

8. Zhou XN, Guo JG, Wu XH, Jiang QW, Zheng J, Dang H, Wang XH, Xu J, Zhu $\mathrm{HQ}$, Wu GL, et al: Epidemiology of schistosomiasis in the People's Republic of China, 2004. Emerg Infect Dis 2007, 13:1470-1476.

9. Hong $Q B$, Huang $Y X$, Zhou XN, Sun $L P$, Xia DH, Xu GK, Tian QA, Tian QA, Zhu JM: Status of schistosomiasis transmission and longitudinal observation on impact of control program in marshland of Jiangsu province. Chin J Schisto Control 2001, 11:34-38.

10. Huang $Y X$, Sun LP, Hong QB, Gao Y, Zhang LH, Gao Y, Chen H, Guo JH, Liang YS, Zhu YC: Longitudinal observation on fluctuation trend of distribution and spread of Oncomelania snails after floodwater in marshland of lower beaches of Yangtze Rive (in Chinese). Chin J Schisto Control 2004, 16:253-256.

11. Li SZ, Luz A, Wang XH, Xu LL, Wang Q, Qian YJ, Wu XH, Guo JG, Xia G, Wang $L Y$, Zhou XN: Schistosomiasis in China: acute infections during 2005-2008. Chin Med J (Engl) 2009, 122:1009-1014.

12. Yuan $H$, Jiagang $G$, Bergquist $R$, Tanner $M$, Xianyi $C$, Huanzeng $W$ : The 1992-1999 World Bank Schistosomiasis Research Initiative in China: outcome and perspectives. Parasitol Int 2000, 49:195-207. 
13. Huang $Y X$, Manderson L: The social and economic context and determinants of schistosomiasis japonica. Acta Trop 2005, 96:223-231.

14. Sun LP, Liang YS, Huang YX, Hong QB, Yang G, Zhang LH, Gao Y, Xie CY: Evaluation on effect of infection control of schistosomiasis in Jiangsu province (in Chinese). Chin J Schisto Control 2008, 20:13-17.

15. Yang GJ, Vounatsou P, Zhou XN, Tanner M, Utzinger J: A Bayesian-based approach for spatio-temporal modeling of county level prevalence of Schistosoma japonicum infection in Jiangsu province, China. Int J Parasitol 2005, 35:155-162.

16. Sun LP, Wang W, Liang YS, Tian ZX, Hong QB, Yang K, Yang GJ, Dai JR, Gao $Y$ : Effect of an integrated control strategy for schistosomiasis japonica in the lower reaches of the Yangtze River, China: an evaluation from 2005 to 2008. Parasit Vectors 2011, 4:243.

17. Zhou YB, Yang MX, Yihuo WL, Liu GM, Wang HY, Wei JG, Jiang QW: Effect of habitat fragmentation on the schistosome-transmitting snail Oncomelania hupensis in a mountainous area of China. Trans $R$ Soc Trop Med Hyg 2011, 105:189-196.

18. Li SZ, Wang YX, Yang K, Liu Q, Wang Q, Zhang Y, Wu XH, Guo JG, Bergquist $R$, Zhou XN: Landscape genetics: the correlation of spatial and genetic distances of Oncomelania hupensis, the intermediate host snail of Schistosoma japonicum in mainland China. Geospat Health 2009, 3:221-231.

19. Yang $\mathrm{K}$, Wang $\mathrm{XH}$, Yang GJ, Wu XH, Qi YL, Li HJ, Zhou XN: An integrated approach to identify distribution of Oncomelania hupensis, the intermediate host of Schistosoma japonicum, in a mountainous region in China. Int J Parasitol 2008, 38:1007-1016.

20. Malone JB, Yang GJ, Leonardo L, Zhou XN: Implementing a geospatial health data infrastructure for control of Asian schistosomiasis in the People's Republic of China and the Philippines. Adv Parasitol 2010, 73:71-100.

21. Zhou XN, Lv S, Yang GJ, Kristensen TK, Bergquist NR, Utzinger J, Malone JB: Spatial epidemiology in zoonotic parasitic diseases: insights gained at the 1st International Symposium on Geospatial Health in Lijiang, China, 2007. Parasit Vectors 2009, 2:10.

22. Scott LM, Janikas MV: Spatial statistics in ArcGIS. Handbook App Spatial Analysis 2010:27-41.

23. Zhang YY, Luo JP, Liu YM, Wang QZ, Chen JH, Xu MX, Xu JM, Wu J, Tu XM, Wu GL, et al: Evaluation of Kato-Katz examination method in three areas with low-level endemicity of schistosomiasis japonica in China: a Bayesian modeling approach. Acta Trop 2009, 112:16-22.

24. Jia XM, Sriplung $H$, Chongsuvivatwong V, Geater A: Sensitivity of pooled serum testing for screening antibody of schistosomiasis japonica by IHA in a mountainous area of Yunnan, China. Parasitology 2009, 136:267-272.

25. Gong J: Clarifying the standard deviational ellipse. Geographical Analysis 2002, 34:155-167.

26. Mitchell A (Ed): The ESRI Guide to GIS Analysis. Redlands: ESRI Press; 2005.

27. Naparus M, Kuntner M: A GIS model predicting potential distributions of a lineage: a test case on hermit spiders (Nephilidae: Nephilengys). PLoS One 2012, 7:e30047.

28. Svensson K, Back E, Eliasson H, Berglund L, Granberg M, Karlsson L, Larsson P, Forsman M, Johansson A: Landscape epidemiology of tularemia outbreaks in Sweden. Emerg Infect Dis 2009, 15:1937-1947.

29. Seto EY, Remais JV, Carlton EJ, Wang S, Liang S, Brindley PJ, Qiu D, Spear RC, Wang LD, Wang TP, et al: Toward sustainable and comprehensive control of schistosomiasis in China: lessons from Sichuan. PLOS Negl Trop Dis 2011, 5:e1372

30. King $\mathrm{CH}$ : Toward the elimination of schistosomiasis. N Engl J Med 2009 360:106-109.

31. Chen MG: Use of praziquantel for clinical treatment and morbidity control of schistosomiasis japonica in China: a review of 30 years' experience. Acta Trop 2005, 96:168-176.

32. Guo J, Li Y, Gray D, Ning A, Hu G, Chen H, Davis GM, Sleigh AC, Feng Z, McManus DP, Williams GM: A drug-based intervention study on the importance of buffaloes for human Schistosoma japonicum infection around Poyang lake, People's Republic of China. Am J Trop Med Hyg 2006, 74:335-341.

33. Zhou XN, Guo JG, Wu XH, Jiang QW, Zheng J, Dang H, Wang XH, Xu J, Zhu $\mathrm{HQ}$, Wu GL: Epidemiology of schistosomiasis in the People's Republic of China, 2004. Emerg Infect Dis 2007, 10:1470-1477.

34. Wang TP, Vang Johansen M, Zhang SQ, Wang FF, Wu WD, Zhang GH, Pan $X P$, Ju Y, Ornbjerg N: Transmission of Schistosoma japonicum by humans and domestic animals in the Yangtze River valley, Anhui province, China. Acta Trop 2005, 96:198-204.

35. Yuan HC: Epidemiological features and control strategies of schistosomiasis japonica in China. Chin Med J (Engl) 1993, 106:563-568.

36. Tsai PJ, Lin ML, Chu CM, Perng CH: Spatial autocorrelation analysis of health care hotspots in Taiwan in 2006. BMC Public Health 2009, 9:464.

37. Hui FM, Xu B, Chen ZW, Cheng X, Liang L, Huang HB, Fang LQ, Yang H, Zhou HN, Yang HL, et al: Spatio-temporal distribution of malaria in Yunnan province, China. Am J Trop Med Hyg 2009, 81:503-509.

38. Yu Q, Dar JR, Wu RF, Bao ZP, Gao JG, Liang YS, Liang YS: Prevalence study on source of infection of schistosomiasis in marshland with infected snails in lower reaches of Yangtze River. Chia J Schisto Control 2010, 22:351-254.

39. Wang $\mathrm{XH}, \mathrm{Wu} \mathrm{XH}$, Zhou XN: Bayesian estimation of community prevalences of Schistosoma japonicum infection in China. Int J Parasitol 2006, 36:895-902.

40. Wang $X H$, Zhou $X N$, Vounatsou $P$, Chen Z, Utzinger J, Yang K, Steinmann $P$, Wu XH: Bayesian spatio-temporal modeling of schistosoma japonicum prevalence data in the absence of a diagnostic 'Gold' standard. PLoS Negl Trop Dis 2008, 2:e250.

\section{doi:10.1186/1756-3305-6-138}

Cite this article as: Yang et al: Spatio-temporal analysis to identify determinants of Oncomelania hupensis infection with Schistosoma japonicum in Jiangsu province, China. Parasites \& Vectors 2013 6:138.

\section{Submit your next manuscript to BioMed Central and take full advantage of:}

- Convenient online submission

- Thorough peer review

- No space constraints or color figure charges

- Immediate publication on acceptance

- Inclusion in PubMed, CAS, Scopus and Google Scholar

- Research which is freely available for redistribution 